\title{
Continuous optical transformations do not elicit unique perceptual descriptions
}

\author{
WILLIAM EPSTEIN and KWONSAENG PARK \\ University of Wisconsin-Madison, Madison, Wisconsin
}

\begin{abstract}
Subjects were exposed to the continuous projective transformations associated with a circle caused to oscillate about its vertical axis. The arc of oscillation was varied and the subjects were required to choose among three alternative descriptions of the appearance of the display during designated segments of the arc of oscillation. There were two principal findings: (1) The subjects divided their responses among descriptions of rigid motion, descriptions of nonrigid motion, and descriptions of concurrent rigid and nonrigid motion. (2) Two indices of perceptual stability showed that the subjects' descriptions did not remain constant for constant optical input. These results are considered in the context of the major theoretical treatments of the perception of structure and depth from motion.
\end{abstract}

A familiar starting point for analyses of perceived depth through motion is the claim that despite the fact that the optical transformations associated with a rigid figure rotating in depth are compatible with both rigid motion in 3$\mathrm{D}$ space and nonrigid motion in the image plane, it is the former description that is invariably reported. This putative state of affairs has set the goal of the analyses: For the proponent of direct realism, the task is to undermine the traditional premise of inherent equivocality of stimulation by discovering information in stimulation that specifies motion in depth (e.g., Todd, 1981); for the constructivist, the task is to discover a computational program that can, at least in principle, compute a unique solution (e.g., Ullman, 1984).

The experiment reported here was not designed to decide between these contrasting approaches. Our aim was more modest: to evaluate the correctness of the claim that motivates these rival efforts. A model of human perception designed to accommodate perceived rigid motion in depth will be decidedly less valuable, if, in fact, the characteristic response to the optical transformations associated with distal rigid motion in 3-D space is somewhat different. Consider two alternatives to the common characterization: (1) When given the opportunity, unbiased observers often report two concurrent events in response to exposure to optical transformations: rigid motion in depth and nonrigid motion in the image plane. (2) When given the opportunity, observers alternately describe rigid motion and nonrigid motion. In both cases, theoretical accounts showing that rigid motion is computable, or that information in stimulation uniquely specifies rigid motion, will have to be qualified in order to retain relevance for the analysis of human perception.

A review of a sample of the literature (e.g., Braunstein, 1976; Gibson \& Gibson, 1957; Johansson, 1964;

The authors' mailing address is W. J. Brogden Psychology Building, University of Wisconsin, Madison, WI 53706.
Johansson \& Jansson, 1968; Koffka, 1935) suggests to us that these two alternatives, that is, concurrent perception and shifting perception, are not uncommon occurrences. However, recognition of this fact has been hampered by investigators' practices of formulating instructions to the subject that emphasize descriptions of configurations and events in 3-D space and of providing ready means only for reporting rigid motion in depth. The former practice creates a strong response bias, and the latter practice artificially constrains the variety of descriptions that can be regularly recorded.

We are aware of only one investigation that was designed to examine concurrent perception. Marmolin (1973a, 1973b) studied the descriptions elicited by a stationary square of light that was caused to expand and contract in both the $x$ and $y$ dimensions at a constant rate of change. When they viewed the square in the absence of countervailing factors, observers reported radial motion, that is, that the square appeared to be advancing and receding cyclically. But Marmolin noted that his subjects routinely underreported motion. The amount of reported motion was less than would be expected from the decoding principle (see Johansson, 1964, 1977), which holds that all of the optical change is "projected out" as motion in depth. This finding led Marmolin to inquire about the subjects' perceptions of size. He found that all subjects reported changes of apparent size (nonrigid motion) as well as motion in depth (rigid motion). The magnitudes of perceived motion in depth and perceived size change were negatively correlated: persons reporting greater amounts of motion reported smaller size change, and vice versa.

The aim of the present experiment was to look for evidence of concurrent perception and shifting perception in a related domain. A luminous circle that oscillated about its vertical axis was presented for inspection, and we sought to establish the perceptual descriptions favored by the observers. From the outset, we considered that three 
descriptions were compatible with the optical input: (1) rigid motion in depth-the circle appears to oscillate in 3-D space, the figure appearing perfectly rigid; (2) changing shape-a deforming shape, all of the change occurring in the frontal plane; that is, no motion in depth is reported; (3) concurrent motion and shape changethe object appears to undergo motion in depth and change in the frontal plane concurrently. In two pilot experiments that preceded the present study, we secured from naive observers their spontaneous verbal descriptions of the oscillating ring under the same viewing conditions that prevailed in the present experiment. In the first of the unpublished experiments, 13 subjects were tested. Eight of these subjects reported only rigid motion; 4 reported only changing shape; 1 reported alternating rigid motion and changing shape. In the second of the unpublished experiments, 14 subjects were tested. Six of these subjects reported only rigid motion; 5 reported only changing shape; 3 reported concurrent rigid motion and changing shape. These observations should allay concern that the data reported here are the result of expectations induced by the procedure we employed in the main experiment. The basic data of the present experiment were the subjects' descriptions of the appearance of the display, expressed by the subjects' responses on a three-alternative forced-choice task.

\section{METHOD}

\section{Subjects}

Twenty University of Wisconsin-Madison undergraduates were paid to serve as subjects. All of the subjects reported having normal or corrected-to-normal vision.

\section{Display}

The source of stimulation was a circular ring formed from a wire $1.6 \mathrm{~mm}$ thick. The diameter of the ring was $15.8 \mathrm{~cm}$ and subtended $4.4^{\circ}$ of visual angle when the ring was oriented in the subject's frontoparallel plane. The wire was coated with saturn yellow fluorescent paint. The viewing distance was $205 \mathrm{~cm}$.

\section{Apparatus}

Two ultraviolet black lights illuminated the ring. One light was located on each side of the ring. The ring was mounted on a verti- cal rod that was attached to a geared motor. Under the control of an Apple II computer, the motor caused the ring to oscillate about the vertical axis at a rate of $28 \% \mathrm{sec}$. Three buttons, appropriately labeled, were provided for manual responses. Responses were recorded by the computer. Two Sonalerts were located directly above the subject's head. One Sonalert emitted a tone of $2900 \mathrm{~Hz}$ and the other a tone of $1900 \mathrm{~Hz}$. Tone duration was $15 \mathrm{msec}$. The two tones were used to demarcate a segment of the arc of oscillation.

\section{Viewing Conditions}

The subject sat in a darkened soundproof booth. Communication with the subject was through an intercom system. The subject used one eye to view the luminous ring through an aperture. While viewing the ring, the subject rested his/her head on a chinrest. The chinrest and aperture were used to minimize depth-perception information due to motion parallax that would arise from head motion and to eliminate depth-perception information due to binocular disparity. The ring was contained in a darkened chamber so that it was the only visible object in the subject's visual field. This mode of presentation eliminated pictorial cues for depth perception.

\section{Design}

In the interest of generality, the degree of oscillation and the location of the probed arc segments were varied. Four different degrees of oscillation were examined: $60^{\circ}, 90^{\circ}, 120^{\circ}$, and $150^{\circ}$. Depending on the degree of oscillation, three to nine $30^{\circ}$ arc segments were probed. Table 1 lists the various combinations of degree of oscillation and arc segment. Each designated arc segment was probed twice within its respective arc of oscillation, once when its forward position demarcated the leading edge of the arc segment and once when its backward position demarcated the leading edge. Accordingly, the number of responses secured for each degree of oscillation was double the number of probed segments. For example, in the case of the $120^{\circ}$ oscillation, each of the seven segments listed in Table 1 was probed twice, resulting in a total of 14 probes.

\section{Procedure}

Each subject was tested on four blocks of 14 randomized conditions (see Table 1). Each trial began with a 1-sec static exposure to the ring in the subject's frontoparallel plane, after which the ring executed three traverses at the selected degree of oscillation. The first traverse on each trial was treated as preparation, and no arc segments were demarcated during the first pass. Over the succeeding two traverses, three or four arc segments were probed. An arc segment was demarcated by the two Sonalert tones. The high tone indicated the start of the arc segment and signaled the subject to attend closely to the display; the low tone indicated the end of the arc segment and signaled the subject to report his or her perceptual

Table 1

Probed Arc Segments for Each Degree of Oscillation

\begin{tabular}{|c|c|c|c|c|c|}
\hline \multirow{2}{*}{$\frac{\text { Oscillation }}{60^{\circ}}$} & \multicolumn{5}{|c|}{ Probed Arc Segments } \\
\hline & $\mathrm{F} 15^{\circ}-\mathrm{B} 15^{\circ}$ & $\begin{array}{l}\text { FP-F30 } \\
\text { FP-B30 }\end{array}$ & & & \\
\hline $90^{\circ}$ & $\mathrm{F} 15^{\circ}-\mathrm{B} 15^{\circ}$ & $\begin{array}{l}\text { FP-F30 } \\
\text { FP-B30 }\end{array}$ & $\begin{array}{l}\mathrm{F} 15^{\circ}-\mathrm{F} 45^{\circ} \\
\mathrm{B} 15^{\circ}-\mathrm{B} 45^{\circ}\end{array}$ & & \\
\hline $120^{\circ}$ & $\mathrm{F} 15^{\circ}-\mathrm{B} 15^{\circ}$ & $\begin{array}{l}\text { FP-F30 } \\
\text { FP-B30 }\end{array}$ & $\begin{array}{l}\mathrm{F} 15^{\circ}-\mathrm{F} 45^{\circ} \\
\mathrm{B} 15^{\circ}-\mathrm{B} 45^{\circ}\end{array}$ & $\begin{array}{l}\mathrm{F} 30^{\circ}-\mathrm{F} 60^{\circ} \\
\mathrm{B} 30^{\circ}-\mathrm{B} 60^{\circ}\end{array}$ & \\
\hline $150^{\circ}$ & $\mathrm{F} 15^{\circ}-\mathrm{B} 15^{\circ}$ & $\begin{array}{l}\text { FP-F30 } \\
\text { FP-B30 }\end{array}$ & $\begin{array}{l}\mathrm{F} 15^{\circ}-\mathrm{F} 45^{\circ} \\
\mathrm{B} 15^{\circ}-\mathrm{B} 45^{\circ}\end{array}$ & $\begin{array}{l}\mathrm{F} 30^{\circ}-\mathrm{F} 60^{\circ} \\
\mathrm{B} 30^{\circ}-\mathrm{B} 60^{\circ}\end{array}$ & $\begin{array}{l}\mathrm{F} 45^{\circ}-\mathrm{F} 75^{\circ} \\
\mathrm{B} 45^{\circ}-\mathrm{B} 75^{\circ}\end{array}$ \\
\hline
\end{tabular}

Note-Each arc segment is specified in terms of the position of the ring in relation to the subject's frontoparallel plane at the beginning and end points of the segment. FP = frontoparallel plane; $F=$ forward from the FP; B = backward from the FP. The paired arcs are proximally equivalent except for direction of motion. 
impression. The temporal interval between the two tones was $1,100 \mathrm{msec}$. The interval between two arc segments (between the end of one segment and the start of the succeeding segment) ranged from $1,600 \mathrm{msec}$ to $6,400 \mathrm{msec}$.

The subject's task was to report the perceptual impression associated with the designated arc segment by pressing one of the three buttons. The subject was instructed to press the left button if the impression was motion in depth alone, the right button if the impression was change in shape alone, and the middle button if it was both motion in depth and change in shape. The subject was urged to report his or her immediate perceptual impression. In addition, the subject was encouraged to respond as quickly as possible after the second tone and to try to base the response exclusively on the impression produced by the designated arc segment. Prompt responding was desirable so that we could match the subject's report with the actual state of stimulation.

\section{RESULTS}

The subject's responses in each category were collapsed across conditions and then the relative frequency for each response category was calculated. The results for each of the 20 subjects are presented in Table 2.

There were large individual differences. Inspection of Table 2 shows that although 11 of the 20 subjects favored motion in depth, no subject used only a single response category. For the remaining 9 subjects, the combined frequency of the other two responses exceeded the frequency of the motion in depth response; 3 of these subjects (C.R.A., T.N.I., and E.I.R.) predominantly reported concurrent motion in depth and change in shape, and 1 sub-

Table 2

Relative Frequencies (in Percent) of Each Subject's Responses for Each Response Category and the Indices of Response Stability

\begin{tabular}{|c|c|c|c|c|c|c|}
\hline \multirow[b]{2}{*}{ Subject } & \multirow{2}{*}{$\begin{array}{c}\text { Total } \\
\text { Responses }\end{array}$} & \multicolumn{3}{|c|}{$\begin{array}{l}\text { Response } \\
\text { Categories }\end{array}$} & \multirow[b]{2}{*}{ WRS } & \multirow[b]{2}{*}{ BRS } \\
\hline & & $\mathbf{A}$ & B & $\mathrm{C}$ & & \\
\hline $\begin{array}{l}\text { S.N.O. } \\
\text { J.A.E. } \\
\text { D.V.A. } \\
\text { D.N.O. } \\
\text { C.C.E. } \\
\text { J.N.O. } \\
\text { D.H.O. } \\
\text { P.T.A. } \\
\text { S.S.U. } \\
\text { S.N.U. } \\
\text { D.U.O. }\end{array}$ & $\begin{array}{l}142 \\
140 \\
137 \\
133 \\
138 \\
137 \\
138 \\
142 \\
141 \\
130 \\
141\end{array}$ & $\begin{array}{l}83 \\
79 \\
78 \\
70 \\
64 \\
63 \\
62 \\
59 \\
58 \\
54 \\
53\end{array}$ & $\begin{array}{r}0 \\
0 \\
22 \\
22 \\
25 \\
14 \\
2 \\
6 \\
6 \\
15 \\
24 \\
6\end{array}$ & $\begin{array}{r}17 \\
21 \\
0 \\
8 \\
11 \\
23 \\
36 \\
35 \\
27 \\
22 \\
40\end{array}$ & $\begin{array}{l}1.41 \\
1.32 \\
1.29 \\
1.67 \\
1.18 \\
1.75 \\
1.69 \\
1.53 \\
1.79 \\
2.22 \\
1.64\end{array}$ & $\begin{array}{l}1.70 \\
1.70 \\
1.82 \\
2.10 \\
1.48 \\
2.84 \\
2.02 \\
2.22 \\
2.66 \\
2.36 \\
2.50\end{array}$ \\
\hline $\begin{array}{l}\text { D.B.E. } \\
\text { M.T.A. } \\
\text { R.B.O. } \\
\text { J.L.U. } \\
\text { I.N.A. }\end{array}$ & $\begin{array}{l}142 \\
125 \\
138 \\
141 \\
141\end{array}$ & $\begin{array}{l}49 \\
49 \\
48 \\
42 \\
39\end{array}$ & $\begin{array}{l}18 \\
15 \\
17 \\
35 \\
36\end{array}$ & $\begin{array}{l}33 \\
36 \\
36 \\
23 \\
25\end{array}$ & $\begin{array}{l}1.66 \\
1.53 \\
1.61 \\
1.86 \\
1.83\end{array}$ & $\begin{array}{l}2.52 \\
2.44 \\
2.08 \\
2.68 \\
2.70\end{array}$ \\
\hline J.H.O. & 144 & 35 & 49 & 16 & 1.51 & 1.88 \\
\hline $\begin{array}{l}\text { E.I.R. } \\
\text { C.R.A. } \\
\text { T.N.I. }\end{array}$ & $\begin{array}{l}132 \\
140 \\
132\end{array}$ & $\begin{array}{r}30 \\
16 \\
8\end{array}$ & $\begin{array}{r}20 \\
3 \\
14\end{array}$ & $\begin{array}{l}50 \\
81 \\
78\end{array}$ & $\begin{array}{l}1.75 \\
1.12 \\
1.57\end{array}$ & $\begin{array}{l}2.26 \\
1.34 \\
2.60\end{array}$ \\
\hline T.N.I. & & 52 & 17 & 31 & & \\
\hline
\end{tabular}

Note-A = motion in depth; $B=$ change in shape; $C=$ both motion in depth and change in shape. WRS = within-replication stability; BRS $=$ between-replications stability. ject favored change in shape. Over all 20 subjects, $52 \%$, $17 \%$, and $31 \%$ of the responses were allocated to motion in depth, change in shape, and concurrent motion and shape change, respectively.

The finding that all subjects reported more than one impression suggests that the subjects' perceptions were unstable, that is, that responses to the same probed arc segment shifted between trials. Two measures of stability were calculated for each subject. The within-replication stability (WRS) index was calculated in the following way. Except for the conditions with the forward(F) $15^{\circ}$ backward(B) $15^{\circ}$ arc segment, in which only two responses (trials) were required, four responses (trials) were required in each condition. (Remember that each condition comprised two distally different but proximally identical arc segments, and each arc segment had two opposite directions of transformations. For the conditions with the $\mathrm{F} 15^{\circ}-\mathrm{B} 15^{\circ}$ arc segment, however, there were no backward or forward locational differences. Accordingly, only two arc segments with the opposite directions of transformation were sampled.) Therefore, all three given response categories could be chosen within one condition. The number of different categories selected within one condition was used as the index of stability of that condition. For example, if a subject chose all three categories within a condition, the WRS index of that condition was 3 . On the other hand, if a subject chose only one category consistently over the four trials within a condition, the WRS index of that condition was 1 . Hence the index of WRS varied from 1 (perfect stability) to 3 (no stability). The WRS indices for conditions with $\mathrm{F} 15^{\circ}-\mathrm{B} 15^{\circ}$ arc segments were calculated in the same way but with a maximum index value of 2 .

The between-replications stability (BRS) index was calculated in a similar way. The number of different categories selected over the replications within one condition, disregarding the order of categories selected within a replication, was used as the BRS index of that condition. Again, the index varied from 1 (perfect stability) to 3 (no stability).

The WRS and BRS indices were then separately averaged over the conditions for each subject. The results are presented in Table 2 . The WRS index varied from 1.12 to 2.22 and averaged 1.59 . The BRS index varied from 1.34 to 2.84 and averaged 2.19. Pearson product correlation between these two indices was $r=.76$, indicating that only slightly over $50 \%$ of the variance was shared by the two indices. The fact that the indices were greater than 1 shows that these optical transformations did not elicit unique descriptions.

To examine the effects of each independent variable on response stability, we first averaged each subject's stability indices for one of the variables across all levels of the other factor. Then we calculated the means for each level of the variable of interest for the 20 subjects. The results are displayed in Figure $1{ }^{1}$ Figure la shows a slight rise and then a slight fall in both BRS and WRS indices with oscillation magnitude. The highest BRS in- 


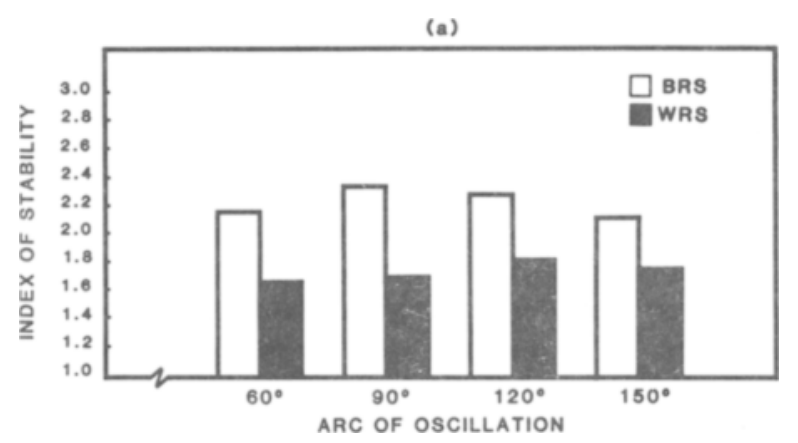

(b)

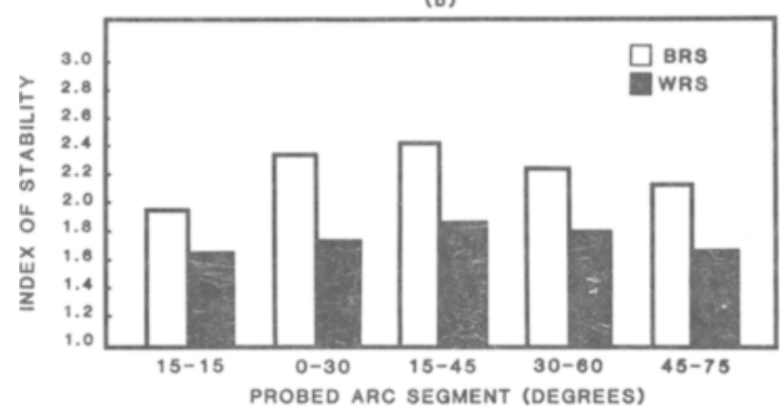

Figure 1. Stability of the subjects' responses in relation to the oscillation magnitude (a) and the probed arc segment (b). (In the conditions crossed with the $15^{\circ}-15^{\circ}$ arc segment, the graphed values are adjusted scores. See Note 1.)

dex was 2.35 at $90^{\circ}$ and the lowest was 2.13 at $150^{\circ}$, whereas the highest WRS was 1.83 at $120^{\circ}$ and the lowest was 1.68 at $60^{\circ}$. However, analyses of variance with repeated measures showed that these variations were not significant $[F(3,57)=1.33, p<.05$ for BRS; $F(3,57)$ $=1.56, p<.05$ for WRS].

Figure $1 \mathrm{~b}$ shows a similar pattern of rise and fall in both BRS and WRS scores with the change of the probed arc segment from $0^{\circ}-30^{\circ}$ to $45^{\circ}-70^{\circ}$. The highest BRS score was 2.45 at the $15^{\circ}-45^{\circ}$ segment and the lowest was 2.15 at the $45^{\circ}-75^{\circ}$ segment, whereas the highest WRS score was 1.88 at the $15^{\circ}-45^{\circ}$ segment and the lowest was 1.68 at the $45^{\circ}-75^{\circ}$ segment. ${ }^{2}$ Again, however, analyses of variance with repeated measures showed no significant effects $[F(3,57)=2.57, p<.05$ for BRS; $F(3,57)=$ $2.70, p<.05$ for WRS].

\section{CONCLUSION}

The experiment yielded three clear findings. (1) When subjects were free to choose among the three descriptions, they divided their responses almost equally between rigid motion in depth $(52 \%)$ and the two descriptions that included nonrigid motion (48\%). (2) Approximately one third $(31 \%)$ of the responses were descriptions of concurrent rigid motion and nonrigid motion. (3) The de- scription selected for any given segment was labile, shifting between trials.

This is not a tidy picture. Nevertheless, these are the facts that a useful model must accommodate. Those who favor plainly computational theories that incorporate the premise that the percipient has a preference for rigid motion will have problems with these data. Even though there is a computable rigid-motion solution for the optical transformation associated with the oscillating ring, nonrigid motion was reported frequently. For the rival theorist, who looks to information in stimulation to specify the percept, the fact that all subjects proffered more than one description presents a challenge.

Computational approaches, and perhaps the informational approach introduced by J. J. Gibson, have as an explicit or implicit premise that the visual system can compute or detect projective correspondence across frames or transformational moments. By employing a smooth, continuously contoured shape, we presented a challenge to the computation or detection of correspondence, and this difficulty may be responsible in part for the high incidence of reports of nonrigid motion. A configuration composed of disconnected points located on the circumference of an imaginary circle might be more amenable to determination of correspondence and less likely to elicit reports of nonrigid motion.

\section{REFERENCES}

Braunstein, M. L. (1976). Depth perception through motion. New York: Academic Press.

Gibson, J. J., \& Gibson, E. J. (1957). Continuous perspective transformations and the perception of rigid motion. Journal of Experimental Psychology, 54, 129-138.

Johansson, G. (1964). Perception of motion and changing form. Scandinavian Journal of Psychology, 5, 181-208.

JoHansson, G. (1977). Spatial constancy and motion in visual perception. In W. Epstein (Ed.), Visual perception: Mechanism and processes. New York: Wiley.

Johansson, G., \& Jansson, G. (1968). Perceived rotary motion from changes in a straight line. Perception \& Psychophysics, 4, 165-170.

KoffKa, K. (1935). Principles of gestalt psychology. London: Routledge \& Kegan Paul.

Marmolin, H. (1973a). Visually perceived motion in depth resulting from proximal changes: I. Perception \& Psychophysics, 14, 133-142.

MARMoLiN, H. (1937b). Visually perceived motion in depth resulting from proximal changes: II. Perception \& Psychophysics, 14, 143-148.

ToDD, J. T. (1981). Visual information about moving objects. Journal of Experimental Psychology: Human Perception \& Performance, 7, 795-810.

ULLMAN, S. (1984). Maximizing rigidity: The incremental recovery of 3-D stimulus from rigid and nonrigid motion. Perception, 13, 255-274.

\section{NOTES}

1. In the conditions crossed with the $\mathrm{F} 15^{\circ}-\mathrm{B} 15^{\circ}$ arc segment, the maximum obtainable stability index was 2 , whereas the maximum was 3 in conditions not crossed with this segment. Therefore, before we computed the marginal means, we adjusted each score in the former conditions by multiplying by 1.335 . The factor 1.335 , rather than 1.5 , was chosen because the actually obtained maximum index from the conditions with the maximum obtainable index of 3 was 2.67 , rather than 3 . 
2. The mean BRS and WRS indices at the $\mathrm{F} 15^{\circ}-\mathrm{B} 15^{\circ}$ segment were excluded from the analyses of variance for the following reasons: Four responses per replication were required in conditions with other segments, whereas only two responses per replication were required in conditions crossed with the $\mathrm{F} 15^{\circ}-\mathrm{B} 15^{\circ}$ segment (for the reason previously mentioned in the text). Consequently, for the WRS index, the maximum obtainable score was 2 , rather than 3 . Thus, the mean WRS score on this segment is not comparable to the mean scores on other segments. (In calculating the marginal means, the scores in the conditions with the $\mathrm{F} 15^{\circ}-\mathrm{B} 15^{\circ}$ segment were adjusted. See Figure 1 caption.) For the
BRS score, similar incomparability may be inferred. Since the WRS index is greater than 1 , we expect more variance in response if four, rather than two, responses are required, which in turn will raise the BRS index as well as the WRS index. Thus, the mean BRS index on the $\mathrm{F} 15^{\circ}-\mathrm{B} 15^{\circ}$ segment can be smaller than those on other segments only because the response variability is limited by the design.

(Manuscript received March 20, 1986; revision accepted for publication July $10,1986$. 